\title{
Redefiniendo la sostenibilidad desde una perspectiva situada: desafíos de museos comunitarios del sur de Chile
}

\author{
Laura Fúquene Giraldo \\ Universidad Austral de Chile, Valdivia, Chile. \\ Email: lcfuqueneg@unal.edu.co \\ Gustavo Blanco Wells \\ Universidad Austral de Chile, Valdivia, Chile. \\ Email: gblanco@uach.cl \\ Karin Weil G. \\ Universidad Austral de Chile, Valdivia, Chile. \\ Email: karinweil@uach.cl
}

\begin{abstract}
Resumen: A partir de la identificación de dimensiones de sostenibilidad, tanto en los ámbitos museológicos como del desarrollo, se encuentran afinidades epistémicas respecto a lo que representa la sostenibilidad para los museos y las comunidades en un plano más amplio. Se analiza cómo museos de pequeña escala, con aciertos y limitaciones, aportan a la sostenibilidad y desarrollo de la comunidad a la que pertenecen. Mediante la caracterización y análisis de tres casos de estudio en la Región de los Ríos, Chile, se identifican dimensiones sinérgicas que desde lo empírico aportan a la discusión teórica sobre la sostenibilidad y valores relacionales como la colaboración, reciprocidad y pertinencia. Se concluye que la proyección de este tipo de museos, se asienta, primero, en la capacidad de ejercer reflexión crítica, segundo, en la construcción de nuevas racionalidades ambientales y culturales y, tercero, en la aparición de nuevos actores sociales cuyas disposiciones afectivas viabilicen la sostenibilidad situadamente.
\end{abstract}

Palabras clave: Nueva museología; autonomía; relacionalidad; cultura material; desarrollo sostenible

\section{Challenges of community museums in southern Chile: Redefining sustainability from a situated perspective}

\begin{abstract}
From the identification of dimensions of sustainability, both in the museological and in the development fields, epistemic affinities are found regarding what sustainability represents for museums and communities in a wider scope. The article analyzes how small-scale museums, with successes and limitations, contribute to the sustainability and development of the community to which they belong. Through the characterization and analysis of three case studies in the Region of Los Ríos, Chile, synergistic dimensions are identified that from the empirical contribute to the theoretical discussion on sustainability and relational values ??such as collaboration, reciprocity and relevance. It is concluded that the projection of this type of museums, is based, first, on the ability to exercise critical reflection, second, on the construction of new environmental and cultural rationalities and third, on the emergence of new social actors whose affective disposition, contribute to make sustainability viable in a situated way.
\end{abstract}

Keywords: New museology; autonomy; relationality; material culture; sustainable development 


\section{Desafios dos museus comunitários no sul do Chile redefinindo a sustentabilidade a partir de uma perspectiva situada}

Resumo: A partir da identificação das dimensões da sustentabilidade, tanto no campo museológico quanto no campo do desenvolvimento, encontram-se afinidades epistêmicas em relação ao que a sustentabilidade representa para os museus e comunidades em um nível mais amplo. Analisa-se como museus de pequena escala, com sucessos e limitações, contribuem para a sustentabilidade e desenvolvimento da comunidade a que pertencem. Através da caracterização e análise de três estudos de caso na Região de Los Ríos, Chile, identificam-se dimensões sinérgicas que contribuem empiricamente para a discussão teórica sobre sustentabilidade e valores relacionais como colaboração, reciprocidade e relevância. Concluise que a projeção deste tipo de museus se baseia, primeiramente, na capacidade de exercer a reflexão crítica, segundo, na construção de novas racionalidades ambientais e culturais e, terceiro, no surgimento de novos atores sociais cujas disposições afetivas viabilizem a sustentabilidade localizada.

Palabras-chave: Nova museología; autonomía; relacionalidade; cultura material; desenvolvimento sustentável.

\section{Introducción}

El rol de los museos en el mundo estuvo unido por mucho tiempo estrictamente a la preservación de la memoria y visión de mundo de las clases aristocráticas (Chagas, 2008), pero en la medida en que las sociedades se fueron transformando, los museos encontraron nuevos desafíos en cuanto a su relación con el entorno humano y ecológico en el que se insertaban (DeCarli, 2003). Ello ha implicado adaptarse y transitar desde una museología tradicional, caracterizada por su importante rol ideológico en la construcción y consolidación de estados naciones, en donde los museos fueron pensados para legitimar estructuras y culturas coloniales en el imaginario colectivo, y avanzar hacia una nueva museología que se caracteriza por cuestionar su rol social y se reconoce a sí misma como una institución al servicio de la sociedad y su desarrollo, pasando por un proceso de democratización, re-significación y apropiación cultural (Chagas, 2008). A este fenómeno se le conoce como Nueva Museología, y se conforma a partir de dos corrientes principales: los ecomuseos y los museos integrales, de los cuales revisaremos sus fundamentos más adelante.

La Nueva Museología está asociada principalmente a la influencia del pensamiento ecologista y los movimientos reformistas democratizadores de las décadas de los 60's y 70's a nivel global y, a dos aspectos en el nivel nacional: la necesidad de mayor descentralización interna, lo que desembocó en la creación de museos regionales (Mostny, 1975); y la crítica sostenida sobre la ausencia de vínculos efectivos con la comunidad, a pesar de la temprana definición acuñada en los estatutos de ICOM el año 1947 y luego perfeccionada y ratificada en 1974 (Marsal, 2012).

La investigación realizada estudia la trama de relaciones que existe entre los museos, las comunidades y los territorios, para analizar cuál es el rol del museo en la actualidad y si este encuentra consonancia con la propuesta de la Nueva Museología, buscando comprender en sus aciertos y limitaciones, si han logrado ser sostenible y de qué forma.

El objeto de estudio es la Red de Museos y Centros Culturales de la región de los Ríos, Chile. Esta es una organización no formal que articula y reúne diversos espacios de casi todas las comunas de la región, y que desde el año 2012 proponen de manera colaborativa y organizada acciones que permitan fortalecer y difundir la historia y memoria local. Cada una de las instituciones que participa de esta Red tiene como objetivo ofrecer un espacio de contención a las comunidades donde están insertas, buscando de diversas formas poner en valor la historia y memoria local. En la actualidad hay más de 20 espacios a lo largo y ancho de la región, constituidos por museos de pequeña y mediana escala, centros culturales, bibliotecas y corporaciones universitarias, públicas, privadas y comunitarias ${ }^{1}$.

El siguiente artículo se centra en tres de los denominados museos de pequeña escala o comunitarios: el Centro Cultural Museo y Memoria de Neltume, el Museo Despierta Hermano de Malalhue y el Museo Escolar Hugo Gunckel, de la Aguada, Corral. Estos casos son de interés debido a su valor social, su historia de confor- 
mación, diversidad de territorios (cordillera, pre cordillera y costa), tipo de institución que representan y enfoque de sus acciones en las comunidades, político-social, cultural y ambiental respectivamente.

Para elaborar una discusión sobre la sostenibilidad y cómo el museo aporta a esta en el desarrollo de las comunidades, es necesaria una contextualización preliminar sobre los conceptos de museo integral y desarrollo sostenible, los cuales componen el marco del horizonte hacia donde están transitando los museos y los enfoques del desarrollo. Ambos son conceptos en proceso de cuestionamiento y construcción permanente.

\section{La Nueva Museología en un periodo de transición}

En medio de la crisis multidimensional que se vivía en la época de los años 60, quienes estaban a cargo de museos cuestionaron su labor, pues consideraban que en una realidad cada vez más globalizada, las exigencias de participación social y la diversidad cultural requerían de un rol más activo de los museos (Dos Santos, 2012), estableciéndose así una corriente teórico-metodológica que se originó en dos reuniones principales (DeCarli, 2003). La primera en el año 1971 en la IX Conferencia Internacional del Consejo Internacional de Museos (ICOM) en Grenoble, Francia, en donde, según Mathilde Bellaigue citada en Laumonier (1993, p.34), nace el concepto de Ecomuseos:

"Tiene como objetivo una comunidad y su desarrollo; esa comunidad está conformada como grupo social heterogéneo, pero unido por las tradiciones, las necesidades, las solidaridades. El ecomuseo no hará de esa comunidad un 'objeto' de estudio, sino el ‘sujeto' de un proceso de autodesarrollo, reconociéndole en primer lugar una cultura, saberes propios, al igual que su derecho a la palabra y a manifestarse como interlocutor válido"

La segunda reunión, la Mesa Redonda de Santiago que tuvo lugar en mayo de 1972 convocada por la Organización de las Naciones Unidas para la Educación, la Ciencia y la Cultura (UNESCO) y organizada por ICOM en Santiago de Chile, donde se abordó la relación de los museos y el desarrollo cultural local y rural de manera interdisciplinar. Uno de los principales resultados fue la definición de un Museo Integral citada en Laumonier, (1993, p. 34)

"El museo es una institución al servicio de la sociedad, de la cual es parte inalienable y tiene en su esencia misma los elementos que le permiten participar en la formación de la conciencia de las comunidades a las cuales sirven y a través de esta conciencia puede contribuir a llevar a la acción a dichas comunidades, proyectando su actividad en el ámbito histórico que debe rematar en la problemática actual; es decir anudando el pasado con el presente y comprometiéndose con los cambios estructurales imperantes y provocando otros dentro de la realidad nacional respectiva”

De esta forma se observa como ante el mismo cuestionamiento surgieron dos propuestas relacionadas: desde la perspectiva europea con los ecomuseos, preocupados por el museo como una herramienta para el autodesarrollo vinculado a su entorno natural, y desde la perspectiva latinoamericana con los museos integrados, preocupados por un rol más político y agente, íntimamente ligado al presente y futuro de la comunidad (DeCarli 2003; Bize, et. al. 2017).

A partir de los encuentros mencionados, se habla de una Nueva Museología, la cual amplía sus horizontes e incluye en su discurso nuevos conceptos, planteando la posibilidad de considerar no un edificio sino un territorio, no una colección sino un patrimonio regional, y no un público sino una comunidad regional participativa (Lacouture, 1994 en DeCarli, 2004). Los principios y declaraciones resultado de la Mesa Redonda de Santiago en 1972, promovieron, principalmente en Europa y Asia, el movimiento de los ecomuseos, y en América Latina, especialmente México, Brasil y más recientemente en Costa Rica, la incorporación de la noción de función social entendida como la necesidad de integrar al museo en el proceso y en la dinámica del desarrollo local (Revista Museo, 2012). A continuación, se hace una revisión sobre el origen de la sostenibilidad en el desarrollo y su implicancia en los museos. 


\section{Origen de las dimensiones de sostenibilidad en el ámbito del desarrollo}

El concepto de sostenibilidad nace en la década de los 70’s en un contexto histórico de preocupación por la crisis ambiental provocada por el sistema económico industrial. Diferentes movimientos ecologistas, ambientalistas y conservacionistas emergieron en la época con posiciones muy diferentes, y que años después, no como resultado de la disolución de diferencias, sino como un proceso de homogenización (Pierri, 2005), convergen en la propuesta del Desarrollo Sostenible mediante el Informe Brundtland, Nuestro Futuro Común en el año 1987 presentado por la ONU.

Para los países industrializados las soluciones se encauzaron bajo la perspectiva de la modernización ecológica (Spaargaren y Mol, 1992), que sugiere que los problemas ambientales se pueden resolver con arreglos institucionales de gestión ambiental y mejoras tecnológicas que pueden prevenir la contaminación o mitigar el daño ambiental (Hajer, 1995). Los países no industrializados reaccionaron de manera reticente a esta versión del desarrollo sustentable. Su mayor preocupación era la pobreza, malnutrición y enfermedades, donde el "no crecimiento" era inaceptable. En consecuencia, en el Informe Founex (Suiza) de 1971, se recogen las preocupaciones económicas y ambientales de los países pobres y se acepta que la expresión "medio ambiente” también incluya aspectos sociales, sin embargo, no se cuestionan en profundidad las causas de la pobreza. Por otra parte, la Fundación de Bariloche (1972), presenta la propuesta latinoamericana donde si se ponen en discusión las bases económicas y políticas del orden actual y proponen alternativas en pos de una sociedad diferente, este modelo tuvo gran impacto en su época y llamó la atención de organismos de la Organización de las Naciones Unidas (ONU), Organización Internacional del Trabajo (OIT) y la UNESCO (Pierri, 2005).

En los documentos e informes acumulados en las sucesivas Cumbres Mundiales sobre el Desarrollo Sostenible, comenzando por Estocolmo en 1972, pasando por la de Rio de Janeiro en 1992, hasta la de Johannesburgo en 2002, la dimensión central claramente es la económica, después la ambiental no como fin en sí misma sino como medio para el crecimiento, y de forma más relegada, la social (Boyer et al, 2016). La dimensión cultural a pesar de haberse mencionado con anterioridad, sólo es considerada como el cuarto pilar para el desarrollo sostenible, a partir del año 2015 en la VII Cumbre de Las Américas celebrada en Panamá.

Con el fin de esclarecer la operatividad de lo que representa la sostenibilidad, tanto en el ámbito museal como en el contexto del desarrollo, se identifican cuatro dimensiones genéricas; social, cultural, ambiental y económica. Esta segmentación si bien se propone como una forma metodológica para facilitar las acciones y análisis a realizar, es una mirada reduccionista que invisibiliza la complejidad de los sistemas de relaciones biológicas, de producción, de información y de poder que las soportan, y que dependen principalmente de las condiciones socio-materiales de cada contexto.

Tras esta breve revisión sobre la transformación de los museos y el origen de las dimensiones de sostenibilidad en el desarrollo, identificamos que, en el contexto nacional, no existen antecedentes sistematizados de la implementación de prácticas o categorías de sostenibilidad. De ahí la importancia de realizar este trabajo en museos que se acercan a la definición del museo integral. Por otra parte, en el ámbito internacional, la sostenibilidad no era un tema de discusión claro y central en los museos, y cuando se consideraba en países como Estados Unidos y Canadá, se le vinculaba a la sostenibilidad financiera o a la gestión ambiental interna en aspectos como eficiencia energética, gestión de residuos, embalaje, entre otras (Sutton, 2015). En menor medida se encuentran algunos casos que incluyen aspectos más relacionados con la función social del museo, como son México, Brasil, Costa Rica (DeCarli, 2004) y Suecia (David, 2011).

Sin embargo, actualmente, organizaciones internacionales como ICOM e Ibermuseos, están promoviendo la implementación de categorías de sostenibilidad tradicionales alineadas con los Objetivos de Desarrollo Sostenible, pero a su vez integrando, en el primer caso, tres deberes morales: satisfacer las necesidades humanas, velar por la justicia social y respetar los límites medioambientales (ICOM, 2018). En el segundo caso, Ibermuseos integra una mirada más transdisciplinaria y crítica en la que problematiza estas mismas dimensiones (Pérez, 2016).

Pasamos a dar cuenta de los desafíos que enfrenta el sector museal al integrarse a sus comunidades de forma sostenible. 


\section{Metodología}

Este artículo es resultado de una investigación social cualitativa, desarrollada por un equipo interdisciplinario con un enfoque interpretativo. El trabajo de levantamiento de información, sistematización y análisis se organizó en cuatro etapas. En la primera, con el propósito de acotar el universo de estudio de 26 museos, se desarrolló una matriz de ponderación que corresponde al desglose de las funciones museológicas que propone la definición oficial de museos ICOM² (Tabla 1). Para su interpretación se utilizó como base el glosario del Comité Internacional para la Museología (ICOFOM, 2010) y se eligieron 4 criterios mínimos (*) a cumplir para ser parte del estudio. La matriz se generó mediante la revisión y análisis de información secundaria sobre la Red de Museos y las instituciones que la componen (base de datos, fichas técnicas existentes y revisión bibliográfica).

Tabla 1.

Componentes de la matriz de ponderación

\begin{tabular}{|c|c|c|c|c|c|c|c|c|c|c|c|}
\hline \multirow{2}{*}{$\begin{array}{l}\text { Institución } \\
\text { Permanente }\end{array}$} & \multirow{2}{*}{$\begin{array}{l}\text { Sin fines de } \\
\text { lucro }\end{array}$} & \multirow{2}{*}{$\begin{array}{l}\text { Sociedad y Desarrollo } \\
\text { (Vinculación) }\end{array}$} & \multirow{2}{*}{$\begin{array}{l}\text { Abierto al } \\
\text { público* }\end{array}$} & \multicolumn{5}{|c|}{$\begin{array}{c}\text { Patrimonio tangible e intangible de la humanidad } \\
\text { (Marcar si tener patrimonio. I: tangible y/o l: intangible) }\end{array}$} & \multicolumn{3}{|c|}{$\begin{array}{l}\text { Ambientación } \\
\text { (Actividades de extension) }\end{array}$} \\
\hline & & & & $\begin{array}{l}\text { Adquierel } \\
\text { Conforma }\end{array}$ & Conserva & Investiga & Comunica & Exhibe & Educación & Estudio & Recreación \\
\hline
\end{tabular}

Fuente: Elaboración propia.

La segunda etapa, constituyó el trabajo de campo y caracterización de las instituciones museales, a partir de las dimensiones de sostenibilidad. Para esta etapa se diseñó un instrumento de levantamiento de datos y su aplicación se realizó a través de entrevistas semi-estructuradas principalmente a los encargados(as) de los museos y centros culturales de la red previamente definidos, en los meses de mayo y junio del 2017.

La tercera etapa, fue la sistematización de los resultados en tres dimensiones principales: territorio, patrimonio y comunidad, partiendo de la propuesta de la Nueva Museología pero sin utilizar categorías cerradas, lo cual permitió elaborar un diagnóstico de la red y la situación actual de las entidades que la conforman.

La cuarta y última etapa, consistió en la selección y análisis de los tres casos de estudio a través de los cuales se problematiza la sostenibilidad, al operacionalizar en preguntas explícitas de un cuestionario, el cruce entre las categorías territorio, patrimonio y comunidad, y las funciones museológicas: institución y gestión, conservación, investigación y conocimiento local, interpretación y mediación, función social. Los criterios para la selección de los museos a analizar en profundidad, además de relevar su valor social en cada uno de sus contextos, atienden a la necesidad de reflejar las gradientes de realidad en los ámbitos ambiental, social, cultural y económico que enfrentan, de modo situado, estas instituciones. La información de los museos y sus características se sintetiza en la Tabla 2 y se ubican geográficamente en la Figura 1.

Tabla 2

Síntesis de los casos de estudio

\begin{tabular}{|c|c|c|c|c|}
\hline MUSEO & Origen & Ambiente & Institución & Temática \\
\hline $\begin{array}{c}\text { Centro Cultural } \\
\text { Museo y Memoria } \\
\text { Neltume }\end{array}$ & Comunitario & $\begin{array}{l}\text { Cordillera } \\
\text { de los Andes }\end{array}$ & Autónoma & $\begin{array}{l}\text { Proceso de } \\
\text { sindicalización } \\
\text { y resistencia } \\
\text { politica }\end{array}$ \\
\hline $\begin{array}{l}\text { Museo Despierta } \\
\text { Hermano. Malalhue }\end{array}$ & $\begin{array}{l}\text { Comunitario } \\
\text { y escolar }\end{array}$ & $\begin{array}{l}\text { Precordillera } \\
\text { de los Andes }\end{array}$ & Autónoma & Interculfuralidad \\
\hline $\begin{array}{l}\text { Museo Escolar } \\
\text { Hugo Gunckel } \\
\text { Corral }\end{array}$ & Escolar & Costa & $\begin{array}{c}\text { Municipal, } \\
\text { Escuela } \\
\text { Pública }\end{array}$ & $\begin{array}{l}\text { Educación } \\
\text { Ambiental }\end{array}$ \\
\hline
\end{tabular}

Fuente: Elaboración propia 
Figura 1.

Mapa de Chile y ubicación de casos de estudio

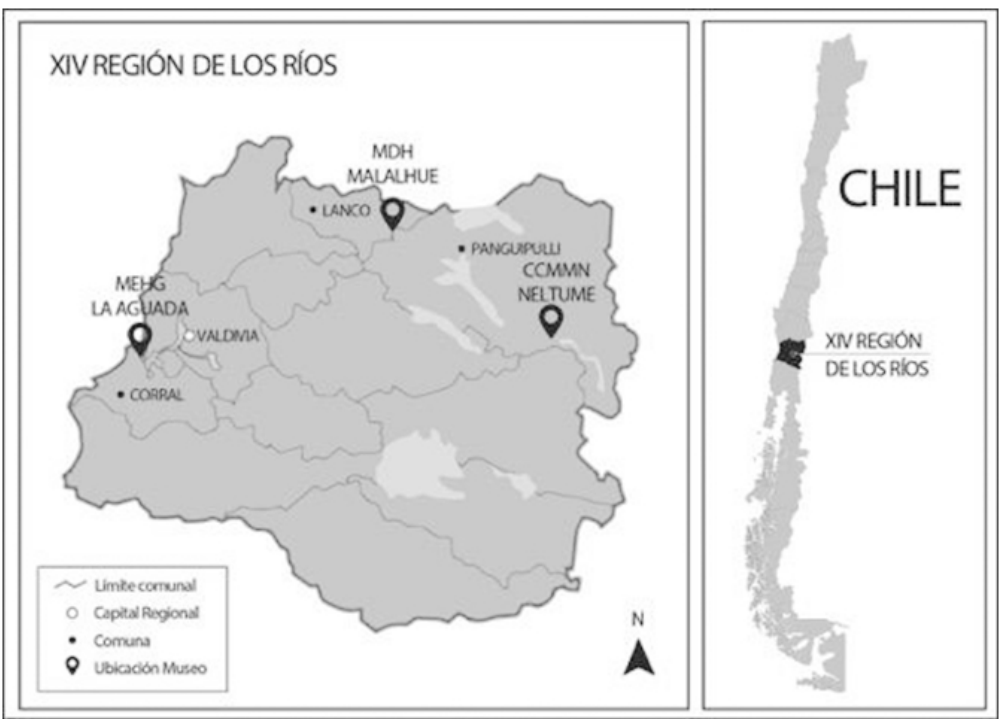

Fuente: elaboración propia.

\section{Resultados}

\section{Dimensiones generales}

Para exponer la realidad de los tres casos de estudio, se presenta a continuación cada uno de ellos haciendo una breve reseña de la institución y su descripción a partir de tres dimensiones generales: Territorio, Patrimonio y Comunidad, las cuales alinean las dimensiones propuestas por la Nueva Museología y por el Desarrollo Sostenible (ver Figura5).

\section{Centro Cultural Museo y Memoria Neltume (CCMMN)}

Como institución funciona bajo la figura legal de Centro Cultural, constituido por el Directorio de la Agrupación Cultural Funcional registrada en la comuna de Panguipulli y su inmueble fue recientemente declarado Monumento Histórico. Respecto a la gestión financiera, los recursos para su operación y actividades se obtienen a partir de tres fuentes: Ministerio de las Culturas, las Artes y el Patrimonio de Chile, El Consejo Nacional de la Cultura y las Artes y un porcentaje mínimo por concepto de corte de entrada (1.000 CLP y aporte voluntario). Respecto a la gestión administrativa, cabe destacar que existe una estructura mixta e intergeneracional conformada por un comité directivo a cargo de las áreas de administración, archivo, museografía, biblioteca comunitaria, extensión y comunicaciones, además de contar con socios(as) locales y honorarios (fuera de Neltume). 
Figura 2.

CCMMN 2018 - Exterior del museo e información general

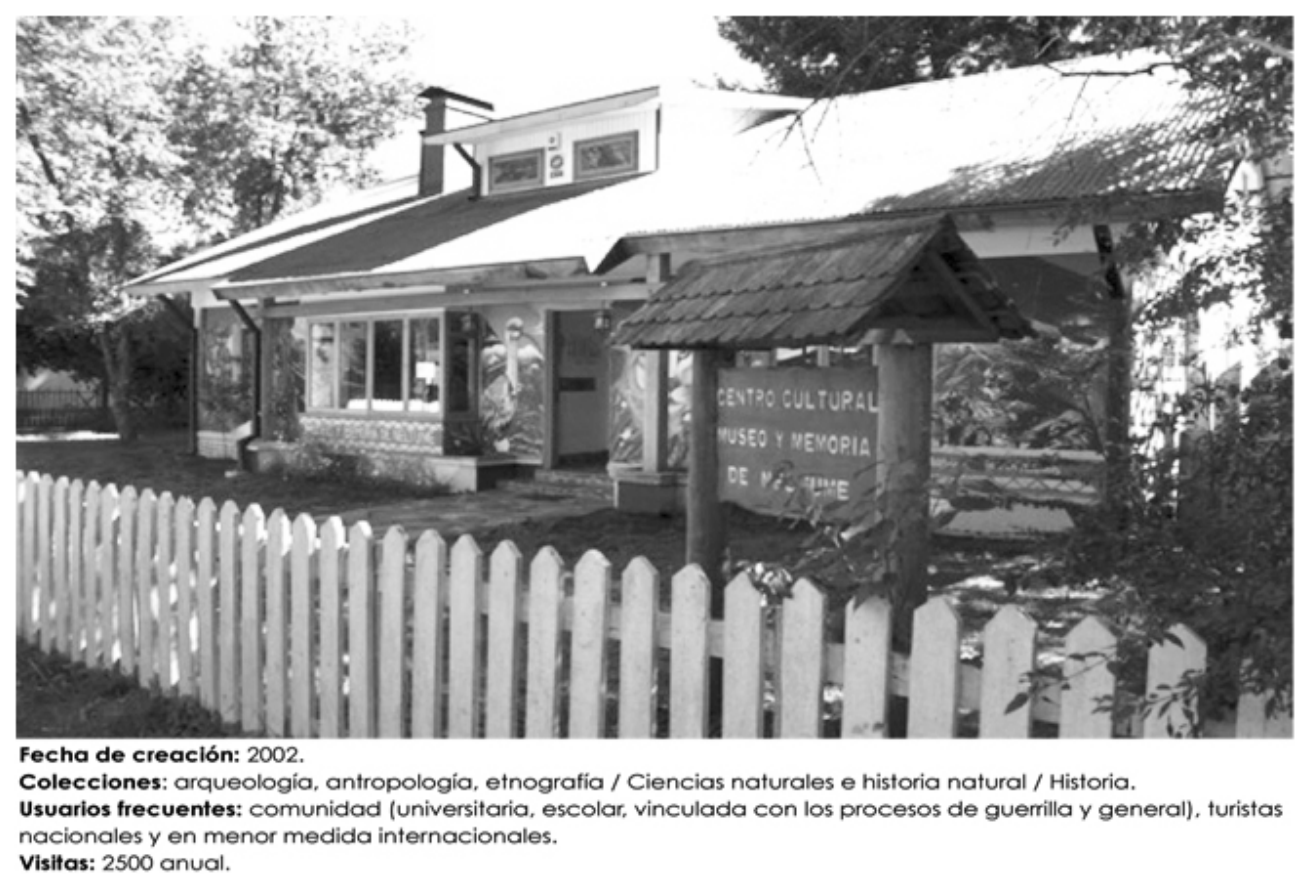

Fuente: Fotografía propia.

Territorio: La localidad de Neltume es predominantemente rural y se emplaza en el sector cordillerano en la comuna de Panguipulli (ver emplazamiento regional en Figura 1). Se trata de una localidad cuya población reciente se fue conformando en torno a labores forestales iniciadas en la década del 30. Posee una población de 2.125 habitantes según el censo del 2002 realizado por el Instituto Nacional de Estadísticas (INE). El territorio de Neltume tiene una larga historia especialmente entre el siglo XX y XXI, asociada a procesos de industrialización, luchas sociales, terrorismo de estado y privatización, que han generado conflictos políticos, económicos y sociales, manifestados en la precarización del trabajo, lo que hace parte de su relato y además afecta de forma directa e indirecta el rol del museo.

Patrimonio: El museo cuenta con un conjunto de colecciones muy diversas, conformadas a partir de objetos personales, entre otros, que fueron donados por la comunidad. El relato presenta un recorrido histórico del territorio cordillerano, iniciado por un breve relato sobre los Mapuche, población originaria del sur de Chile y Argentina; le sigue el proceso de industrialización del territorio para la explotación del bosque nativo; los procesos de sindicalización y organización política; la conformación y experiencia del Complejo Forestal y Maderero Panguipulli (COFOMAP); el golpe de Estado y violaciones a los derechos humanos en dictadura (1973-1990); hasta su vinculación con las luchas actuales por el agua y la reivindicación de las tierras por los pueblos originarios.

Comunidad: Este museo establece algunas relaciones estatales, pero principalmente comunitarias, vinculando organizaciones de diversos tipos y temáticas, lo que le brinda una amplia red de apoyo logístico para la generación de contenidos y programación pertinente y de actualidad. La comunidad se hace partícipe principalmente en las diversas actividades que allí se realizan, más que en la gestión misma. Hay una importante participación de los sobrevivientes de la guerrilla y antiguos trabajadores que ya no viven en la localidad. Esta institución además de ser museo, es centro cultural y biblioteca, diversificando así sus actividades y espacios, logrando fortalecer a través de la educación el lazo con la comunidad. En dos ocasiones (año 2013 y 2018) han sido víctimas de robo y ataques incendiarios, frente a los cuales la comunidad ha manifestado su apoyo al museo y rechazo a tales episodios. 


\section{Museo Despierta Hermano, Malalhue (MDH)}

Como museo funcionan al alero de la Agrupación Cultural Malalhue Siembra, espacio que alberga la radio y biblioteca comunitaria. El edificio es propiedad del Servicio de Vivienda y Urbanismo de Chile (Serviu). El conjunto de colecciones y soportes para su exhibición, han sido facilitados por la comunidad y son responsabilidad de la fundadora del museo, ya que aún no han constituido una personería jurídica. En relación a la gestión financiera, el museo no obtiene recursos económicos de ninguna entidad, sólo cuenta con el aporte voluntario de los visitantes, siendo mínimo. Por otro lado, la municipalidad designa media jornada laboral de una educadora intercultural, para dedicarla al museo como guía. Respecto a la gestión administrativa, La fundadora junto a la educadora, equipo del museo, mantienen buena comunicación y la toma de decisiones se hace de manera horizontal.

Figura 3.

MDH 2017 - Interior del museo e información general

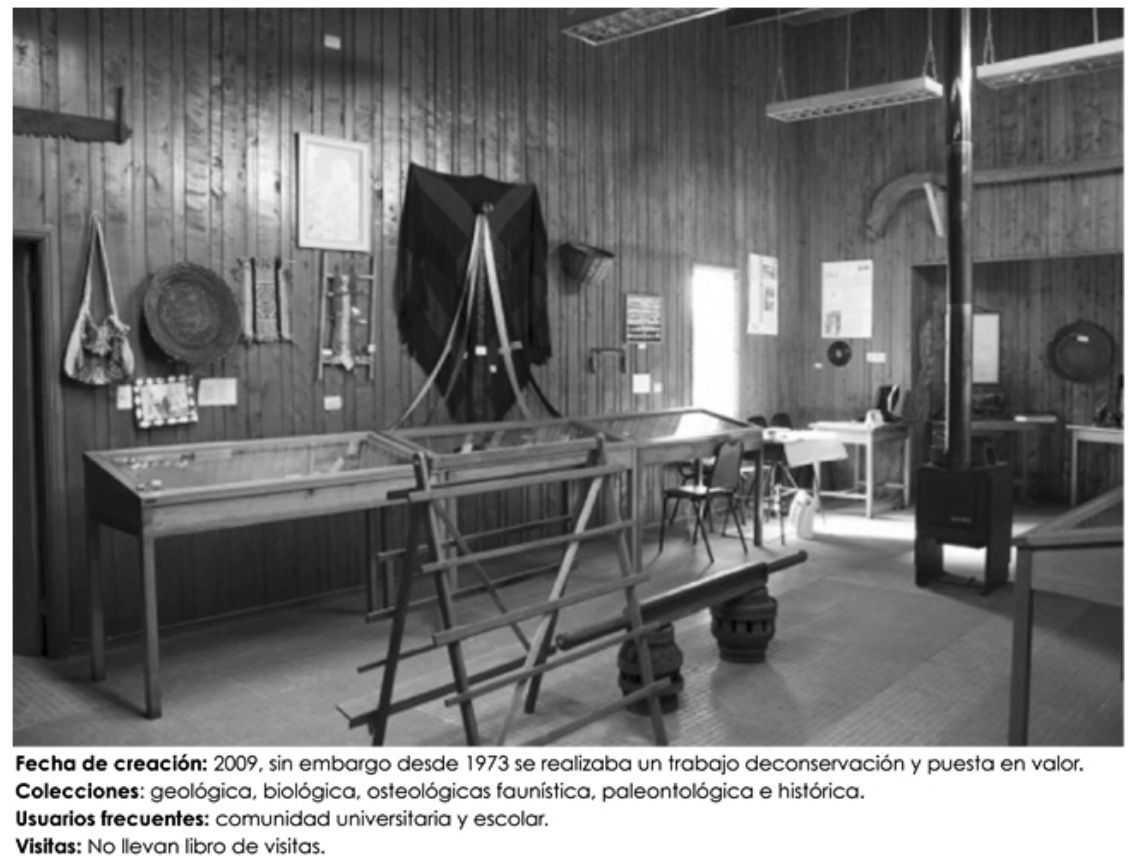

Fuente: fotografía propia.

Territorio: La localidad de Malalhue antiguamente llamada Eulafque (dos Ríos), es una localidad rural de la comuna de Lanco emplazada en un amplio valle entre cursos de agua, bosques nativos, plantaciones agrícolas y predomina la población mapuche (ver emplazamiento regional en Figura 1). Para esta localidad se estima una población cercana a 3.150 habitantes, según datos de la I. Municipalidad de Lanco.

Patrimonio: El museo cuenta con colecciones arqueológicas, históricas, etnográficas y filatélicas. Cada una de estas colecciones se compone por distintos tipos de objetos donde destacan piezas cerámicas, textiles y artefactos propios de la historia moderna del pueblo de Malalhue, donadas por las comunidades locales. Éste nace y se caracteriza por preservar el conocimiento de la cultura mapuche y, según declara su fundadora, en oposición a la educación formal escolar que busca la homogeneidad cultural a nivel nacional.

Comunidad: El museo establece principalmente relaciones con la comunidad indígena del territorio, pues como lo expresan sus encargadas, este museo se caracteriza por haber surgido de la comunidad y por tener el registro de cada objeto con la historia de a quién perteneció y cómo llegó hasta allí. Desde los orígenes del museo y hasta la fecha, ha sido fundamental contar con el apoyo, reconocimiento y soporte de las autoridades 
ancestrales mapuche. La comunidad escolar y local ha concebido la colección y contenidos del museo, pero no hay participación explícita de ésta y las autoridades comunales en su gestión.

\section{Museo Escolar Hugo Gunckel, La Aguada (MEHG)}

El museo se encuentra emplazado en el edificio de la Escuela Rural de La Aguada, dependiente de la Ilustre Municipalidad de Corral, ubicada en una zona de riesgo por remociones en masa, y es considerada un área de protección por el estero que la colinda. Respecto a la gestión administrativa, actualmente es desarrollada por un ex alumno y profesor, quien está a cargo del espacio y sus colecciones, sin embargo las decisiones dependen de la Directora de la Escuela y a través del Departamento de Educación Municipal (DAEM) se obtienen los recursos para las actividades.

Figura 4.

MEHG 2017 - Interior del museo e información general

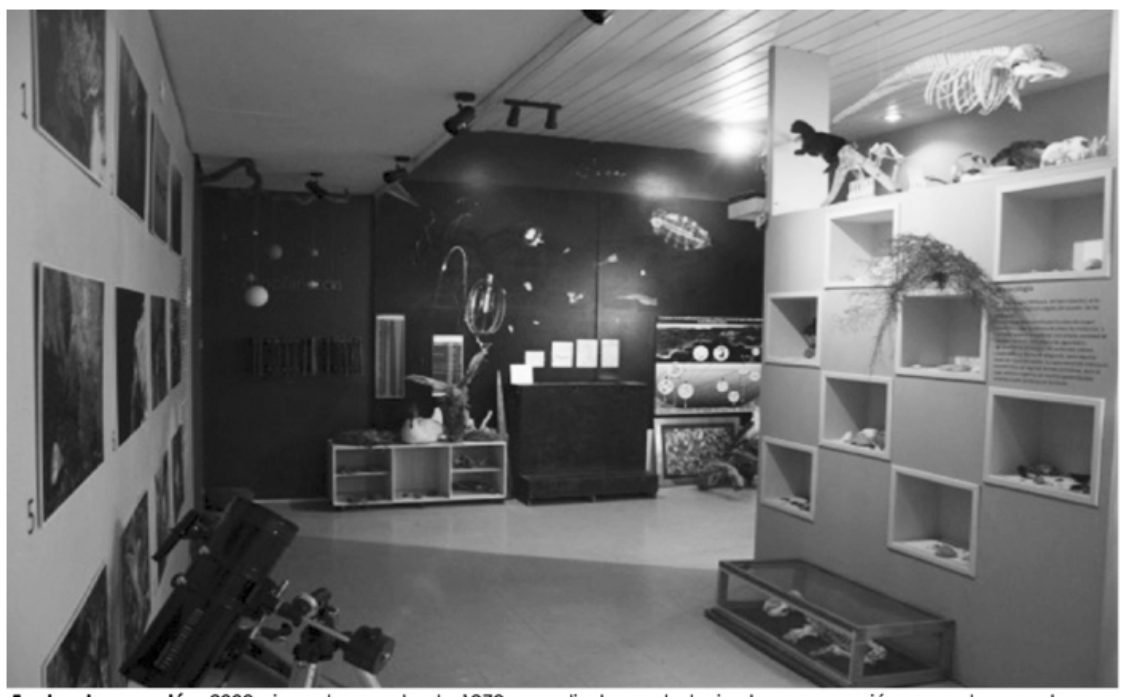

Fecha de creación: 2009, sin embargo desde 1973 se realizaba un trabajo deconservación y puesta en valor. Colecciones: geológica, biológica, osteológicas faunística, paleontológica e histórica. Usuarios frecuentes: comunidad universitaria y escolar. Visitas: No llevan libro de visitas.

Fuente: fotografía propia.

Territorio: La Aguada pertenece a la comuna de Corral en la desembocadura del río Valdivia. La comuna tiene una superficie de 766,7 km2 y una población de 5.463 personas (ver emplazamiento regional en Figura 1). El sector de La Aguada se conforma con una población mucho menor a partir de la llegada de la empresa siderúrgica "Los Altos Hornos" en el año 1906, lo que provoca el arribo de mano de obra calificada, personas y familias, quienes se instalaron en ese territorio. Desde entonces a la fecha, la localidad ha sufrido grandes transformaciones y migraciones sujetas principalmente al desarrollo de actividades productivas. En la actualidad los habitantes del lugar, se dedican principalmente a la pesca artesanal y a trabajos de temporada. Si bien esta zona es considerada urbana, presenta características propias de la ruralidad dada su condición de segregación social y territorial del resto de la comuna(Basso et al., 2010).

Patrimonio: El museo exhibe colecciones que aluden a la biodiversidad de la costa de Corral. Entre ellas hay colecciones y muestras geológicas, así como muestras biológicas, osteológica, faunística, paleontológica e históricas. El origen del museo y las colecciones se remonta al año 1973, obtenidas por el profesor Miguel Hernández en salidas a terreno de reconocimiento del territorio y donaciones hechas por pescadores de la zona. La principal característica es el rescate del patrimonio natural como un recurso pedagógico. Exponen sus colec- 
ciones en vitrinas con sus fichas informativas respectivas y realizan acciones de taxidermia y conservación preventiva, también cuentan con el apoyo de una profesional en museología que dirige el taller de museología científica.

Comunidad: El museo establece principalmente relaciones comunitarias. Por medio de los niños se transmiten conocimientos a los padres, quienes también participan en las actividades y talleres que se realizan en el museo. Se manifiesta una estrecha relación con la comunidad expresada en hechos como que los ex alumnos, frecuentemente regresan al museo y envían donaciones como minerales y piedras preciosas. La creciente demanda del público está motivando el acceso al museo de manera independiente a la escuela, para abrir el espacio de manera permanente a todo público.

\section{Dimensiones sinérgicas}

Dada la complejidad de las relaciones que se manifiestan y observando que una división analítica exclusivamente centrada en las dimensiones generales de la sostenibilidad museológica (Patrimonio, Territorio y Comunidad) puede ser restringida, se amplía la discusión a partir de dimensiones sinérgicas y sus características en cada caso. Estas dimensiones resultan en la intersección de dos o más de las dimensiones generales y visibiliza las acciones que allí tienen lugar. Esta propuesta analítica se representa esquemáticamente en la Figura 2.

Figura 5.

Esquema de propuesta dimensiones analíticas de la sostenibilidad

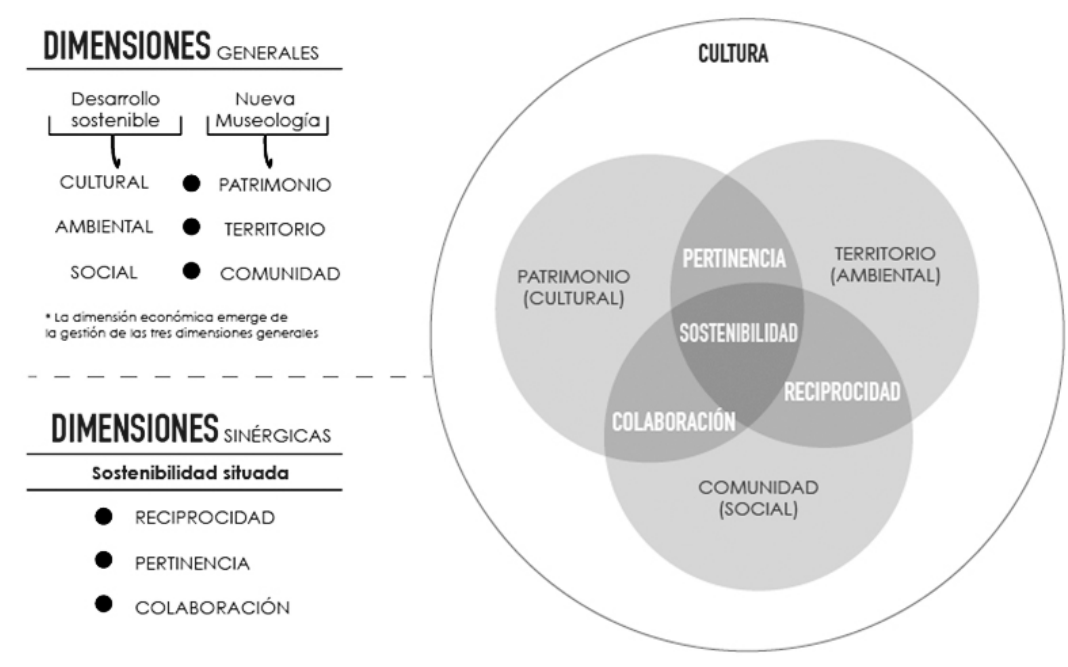

Fuente: elaboración propia.

Esta propuesta de análisis es consonante con las teorías de la Sinergia Humana (Mallman, et. al., 1978) y el Desarrollo a Escala Humana (Max-Neef, et. al., 1986), las cuales surgen en la búsqueda de un desarrollo que, además de atender las necesidades de subsistencia, atiende las necesidades del ser.

\section{Reciprocidad: Sinergia comunidad - territorio}

La reciprocidad representa, en este caso, un modo de sinergia a través de la forma en la que la comunidad se relaciona con su entorno: cómo lo entiende, qué representa la naturaleza para sí y como esto se estimula, 
potencia y/o visibiliza en el museo al ser un espacio que se inserta en el territorio. A continuación, se analiza esta relación para cada uno de los museos del estudio.

CCMMN: El proceso de conformación de la comunidad de Neltume obedece principalmente a factores productivos, ya que el habitar de este territorio ha sido a partir de la explotación de recursos naturales; la tierra, la madera y el agua, por lo tanto, el rol de la naturaleza en este caso, es el de recurso económico y de sustento, que ha configurado los sistemas sociales y culturales, como también los discursos y luchas que allí han tenido lugar. En relación a esta dimensión discursiva, su encargada lo describe de la siguiente forma:

"Neltume es un museo que se caracteriza por escribir lo que la gente ha dicho, tal y como lo dicen, como lo piensan y como lo sienten, nosotros no ponemos las palabras desde otro ámbito más ilustrado, sino que solamente como la gente lo habla y lo siente, se transmite (Actor local del museo, Neltume, mayo 2017”.

MDH: El territorio de Malalhue se desarrolló en el siglo XX, en torno a la estación del tren e históricamente ha estado conformado principalmente por comunidades indígenas mapuche, quienes tienen un fuerte vínculo de inter-dependencia con la naturaleza, por tanto, la forma en que se representan y coexisten es atávica a su cosmovisión en el más amplio de los sentidos. Esta cosmovisión se incorpora en un país con el cual tiene grandes tensiones, ya que funciona bajo dinámicas que homogenizan el conocimiento y entendimiento del entorno como un recurso a ser explotado. Dicha situación es la causa por la cual nace este museo, pues a partir de las tensiones expresadas en aulas y grupos escolares, se hicieron actividades para poner en valor la diversidad intercultural del territorio.

MEHG: La relación que establece la comunidad de La Aguada con la naturaleza es dicotómica, en cuanto por una parte es productiva y por otra, obedece a la necesidad de conservación y restauración del hábitat, el cual fue deteriorado por la instalación de los altos hornos siderúrgicos y otros procesos industriales de principios del siglo XX. Para la comunidad escolar, la relación es académica y se caracteriza por la investigación y reflexión en cuanto al impacto antrópico sobre el paisaje, relevando los conocimientos científicos y tradicionales de la flora y fauna local con fines de preservación. Lo interesante en este caso, es reconocer el incentivo a la relación de la cosmovisión mapuche con el paradigma occidental y racional de la comunidad académica, para profundizar en la relación de interdependencia con la naturaleza y promover el bienestar ambiental y humano, que conforman el territorio y la comunidad en general.

En lo expuesto sobre la relación de reciprocidad entre la comunidad y el territorio, se puede identificar en el caso de Neltume, que la relación es centrada en el ser humano el cual tiene poder sobre la naturaleza, para su explotación como recurso y sustento. En el caso de la Aguada, la relación también parte del ser humano, pero contrario al caso anterior, el enfoque se centra en la necesidad de relevar la importancia de la naturaleza en la vida humana. Sin embargo, en el transcurso del tiempo, gracias a las demandas sociales en la actualización de las identidades y los procesos de hibridación de diferentes formas del ser (Leff, 2000), ambos casos están transitando hacia el entendimiento de la interdependencia, la creación de conciencia y la realización de acciones frente al inminente deterioro del ambiente. Por otra parte, en el caso de Malalhue, donde no existe una división entre ser humano y naturaleza, pues se es parte de ella, y desde esta forma sistémica de entenderla, se conciben la cultura y significación de los objetos que la representan, fomentando la complementariedad y las prácticas de cooperación inter-étnicas para el entendimiento integrado de los recursos naturales.

\section{Colaboración: Sinergia comunidad - patrimonio}

Esta sinergia representa la forma en que las personas de la comunidad han traducido el entendimiento de su entorno, pudiendo determinar los objetos o elementos del mismo, que son relevantes de conservar y su respectivo argumento. Esta es quizás la dimensión más compleja y que genera más tensiones en el ejercicio museal, debido a que los procesos de interpretación y mediación, siempre van a representar a una parte limitada de la comunidad.

CCMMN: El guion museográfico en este caso se construye a partir de los relatos de memoria e historia oral. Son estas las que contextualizan y ponen en valor los objetos que componen la colección en exhibición, 
relevándose a partir de su valor simbólico en cuanto constituyen la evidencia material de los testimonios de vida de las personas de la comunidad, y no por la excepcionalidad de su fabricación, materiales, estética o valor económico. La necesidad de exponer a partir de elementos de interpretación es siempre un riesgo. La memoria, a diferencia de los hechos históricos, expone las vivencias subjetivas en conflicto, generando, muchas veces, encuentros y desencuentros entres quiénes las rememoran.

MDH: De manera similar al CCMMN los contenidos y relatos del museo, se han construido a partir de la memoria e historia oral y los objetos significativos, que la comunidad ha relevado y contribuido para la conformación del museo. Se han realizado cabildos ${ }^{3}$ del agua, la madera y los árboles, en los cuales las personas de la comunidad conversan y reconstruyen una secuencia histórica de la transformación del paisaje que hoy ya no existe, memorias que quedan escritas y a disposición de la comunidad. “(...) así es como se sustenta este museo, nosotros no vamos escarbando, ni comprando, ni pidiendo, las cosas llegan solas” (Actor local del museo, Malalhue, mayo 2017). Como lo exponen los actores relevantes en la constitución y actual gestión del museo, la mayoría de las colecciones fueron donadas debido a la confianza entre el museo y la comunidad, en su interés por preservar sus memorias, característica principal de este museo.

MEHG: En este caso, la construcción museográfica surge desde lo práctico y experiencial, por medio de salidas a terreno de los estudiantes, se interactúa con pescadores artesanales y buzos, entre otros, relacionando el conocimiento originario con el conocimiento científico. A través del taller de taxidermia y museología con un enfoque transdisciplinario y holístico, la experiencia práctica es la forma de conocer las especies y el ecosistema en el que se insertan, destacando su valor endémico y generando conciencia sobre el impacto del ser humano en la naturaleza. En este caso, las colecciones son vida en sí mismas, se trata de la interacción con especies naturales en el presente y a partir de ahí, comprender el pasado y las causas que le generaron posibles transformaciones.

De acuerdo a nuestra propuesta analítica la relación entre la comunidad y el patrimonio, se identifica que la colaboración es fundamental para la gestión y significación del patrimonio natural y cultural de los territorios, pues a través de las historias familiares se generan vínculos de cercanía e identidad entre el museo y la comunidad, dos cosas esenciales para su sostenibilidad (De Carli, 2004). Sin embargo, también se identifica una tensión implícita en las relaciones, los contenidos y su misma proyección, así como su inestabilidad al depender principalmente del compromiso de sus fundadores.

\section{Pertinencia: Sinergia patrimonio - territorio}

Esta sinergia representa la vigencia de lo que se pone en valor en el museo y su agencia en la realidad actual del territorio, con determinados fines políticos, ambientales, educativos y/o de preservación.

CCMMN: El museo, aunque no inicia actividades concretas que determinen una postura crítica frente a conflictos actuales en el territorio, sí participa cuando lo convocan como colaborador. Por ejemplo, en el caso de la muerte de la activista Macarena Valdés y el Movimiento por las Aguas y los Territorios (MAT), ambos en contra de mega proyectos industriales y eléctricos, el museo facilitó su espacio para el encuentro, la discusión y difusión de la situación, lo que le permitió acercarse a la comunidad y participar activamente, a pesar de tener algunos socios del museo detractores de su participación por miedo a represalias.

MDH: Frente a plantaciones forestales que han intentado ocupar territorios sagrados, el museo ha apoyado las iniciativas de las comunidades para resistirse. En los cabildos que convoca, los cuales generan conciencia sobre la importancia del cuidado y el sentido de pertenencia por el territorio, el museo no genera acciones que vinculen activamente el conocimiento histórico con situaciones actuales, lo que representa una pérdida de interés en la comunidad local y joven, de acuerdo a lo mencionado en entrevistas realizadas a personas locales.

MEHG: El museo a través de su enfoque ecosistémico y de la puesta en valor del patrimonio natural, analiza y da cuenta de los impactos ambientales de la industria y los procesos políticos y económicos del entorno, creando conciencia y pensamiento crítico frente a estas situaciones, como lo expone el profesor encargado: 
"En una ocasión hubo un derrame de aceite en la pesquera, que afectó todo el litoral de la bahía, ahí, por ejemplo, salimos a terreno, hicimos muestreos y lo ligamos con la clase de ciencias naturales (...) este cuestionamiento desde nosotros, pasando por los niños, hace que llegue hasta las casas” (Actor local del museo, La Aguada, marzo 2018).

A través de su metodología se hace del aprendizaje, una experiencia viva y del museo un recurso didáctico, que posteriormente trasciende a denuncias públicas, exponiendo los resultados de investigaciones en Juntas vecinales y reuniones de Padres, e incluso articulándose con investigaciones universitarias.

Finalmente, la pertinencia como sinergia, está más asociada con la propuesta de Museo Integrado, en cuanto a exponer las diversas posturas o realidades de conflicto en el contexto local, donde la educación juega un rol fundamental respecto a cuál historia tienen acceso las nuevas generaciones. Así mismo, mientras más conexiones les permita establecer, entre el presente y el pasado, más posibilidades de proyectarse en el futuro podrá tener. Enmarcado en este contexto, los museos constituyendo espacios de convergencia y diálogo de saberes (Quijano, 2000), generando conciencia crítica de su realidad y empoderamiento para resistirse a modelos globalizados.

A partir de los resultados y su análisis, se da cuenta de la génesis de cada museo y las diferentes formas de su devenir en el tiempo, relevando qué cosas han caracterizado su sostenibilidad hasta ahora y qué cosas le dificultan su proyección en el futuro, lo que permite identificar algunas correlaciones respecto al desarrollo y la sostenibilidad.

\section{Discusión: hacia una definición situada de la sostenibilidad}

La discusión del artículo se elabora entorno a la definición y los aprendizajes de la sostenibilidad situada, y las brechas y oportunidades que se pueden identificar en ella. Destacando que en las relaciones socionaturales se encuentran valores que propician la sostenibilidad, pero que, a su vez, están insertas en un sistema económico que actualmente continúa teniendo gran influencia y determinación en las realidades, razón por la cual la dimensión cultural es clave para la creación de nuevas alternativas al desarrollo.

Aun cuando la sostenibilidad, en su dimensión discursiva, es cada vez más asimilada por la racionalidad económica, de manera empírica sus dimensiones se están arraigando en el ámbito local a través de nuevas racionalidades ambientales (Leff, 2000), las cuales tienen una suerte de sincretismo, pues no surgen del acuerdo, sino de la coexistencia en el proceso de enfrentar las dificultades. Así, el proceso de adaptación y asimilación de las situaciones actuales, encuentran en el museo, un espacio para la regeneración de nuevos significados, valores y acciones culturales, en relación con la naturaleza y apropiación social de la ciencia y la tecnología.

De esta forma vemos la contribución de las relaciones que existen entre las diferentes dimensiones de la sostenibilidad enraizada y situada, al estudio fragmentado que plantea la sostenibilidad "genérica". Al analizar cada caso museal, por medio de dimensiones genéricas y dimensiones sinérgicas, se puede observar que la segmentación tiende a objetualizar aspectos que tratan de sujetos, cuyas relaciones no tienen límites claramente definidos, por el contrario, son entidades vivas y orgánicas que se transforman de manera recíproca.

Así, las dimensiones genéricas como aquellas que describen lo que existe, y las dimensiones sinérgicas como expresión endógena de las acciones locales (Max-Neef, 2004), plantean una correlación que desafía al sistema dominante que es incapaz de asignar valores sin reducirlos a valores económicos, en el que los seres humanos son consumidores o capital y la naturaleza son recursos explotables. Como contrapunto podemos comprender estos fenómenos desde una mirada relacional, enfocada en los procesos y las dimensiones integradas (Boyer et. al., 2016), lo que nos revelaría el repertorio amplio de acciones que permiten hacer frente a problemas cotidianos que constituyen el tejido básico de la vida. Esto es la noción de "trabajo significativo" según el autor Ezio Manzini (2015) quien releva el valor y poder de la colaboración, como una condición necesaria para "conseguir que suceda algo" y para que la gente pueda desempeñar un papel activo en la construcción del futuro. 
Por lo tanto, la pequeña escala y la interconexión que caracteriza estos museos, les permite arraigarse en un lugar de manera más profunda. Al mismo tiempo, al estar tan interconectados, son entidades abiertas que dejan de estar aisladas y se convierten en parte de las prácticas culturales, que en un mediano plazo pueden regenerar el tejido social-ambiental local, y en un plazo mas extendido, desarrollar estrategias que empoderen y favorezcan la resiliencia de las comunidades, frente a las amenazas y problemas externos. Sin embargo, esta interconexión y construcción dinámica de sentidos compartidos, pueden proponerse como factores o condiciones de riesgo en el contexto y ser problematizada en las dimensiones de sostenibilidad situada.

De acuerdo a las características identificadas, este tipo de museos se pueden proyectar como museos vivos, integrados y sostenibles, en cuanto más estrecha su relación con las comunidades, que no son abstractas ni retóricas, sino un entramado de seres humanos y no humanos en ambientes concretos. Personas y objetos son mutuamente constituidos, y en esa interacción, se generan otros procesos sociales creativos que dan forma al sitio social en que tiene lugar la vida con una carga histórica, en la que el valor es atribuido al objeto, por los significados que contiene y su capacidad para evocarlos. El Museo Integrado, se convierte en ese sitio social, transformando, su rol tradicional a través de su función social y así "en la formación de la conciencia de las comunidades [...] puede contribuir a llevar a la acción a dichas comunidades” de manera regeneradora, "proyectando su actividad en el ámbito histórico que debe rematar en la problemática actual” (Dos Santos, 2012).

\section{Dificultades y oportunidades en la sostenibilidad situada}

En la investigación llevada a cabo en estos museos, las personas a cargo que fueron entrevistadas, manifestaron que la insostenibilidad de sus espacios se debía principalmente a sus limitaciones económicas y al bajo o débil respaldo institucional especialmente de los gobiernos locales, pero a su vez resaltaron el valor de la autonomía del museo para la toma de decisiones. Al analizar los resultados se pudo observar que ser autónomo institucionalmente o depender del Estado, no garantiza la sostenibilidad de los museos, pero si lo hace la capacidad de gestión con la que cuenten.

Por ejemplo, el museo de Malalhue tiene completa autonomía, pero no cuenta con la capacidad instalada para gestionar ni ejecutar recursos. El museo escolar de la Aguada, esta al alero de una institución gubernamental con personal capacitado y dispuesto a gestionar, pero los intereses de la institución y su burocracia tienen otras prioridades que no le permiten aprovechar su potencial.

El caso del museo en Neltume, por otra parte, cuenta con una combinación de autonomía y capacidad de gestión, en una red de apoyo que vincula entidades gubernamentales, comunitarias y equipos profesionales, que han facilitado la obtención de recursos para su funcionamiento y el desarrollo de mecanismos de participación para la comunidad en diferentes niveles (usuario, colaborador, creador de colecciones) y grupos generacionales, contribuyendo al empoderamiento y desarrollo de la comunidad, con acciones pertinentes a las situaciones locales. Sin embargo, dicho proceso de articulación, también representa grandes retos y conflictos en la selección de las partes que participan, debido a la sensibilidad y complejidad política que representa este museo, y también los sentimientos y las experiencias traumáticas que esto despierta en los actores y familias involucradas.

Lo expuesto demuestra en primer lugar, cómo la posibilidad de proyectar la sostenibilidad de los museos de pequeña escala, se encuentra extremadamente vinculada a las motivaciones de cada encargada(o), siendo este líder del proceso, en función del territorio, las necesidades y capacidades de la comunidad, lo que a su vez lo hace frágil. Por una parte y por otra, implica democratizar el acceso al patrimonio natural y redistribuir, a escala local, la carga ecológica (Quiroga, 2012). Así mismo, al potenciar mayores niveles de participación y autonomía en la toma de decisiones, a través de relaciones que le permitan a la comunidad garantizar su sustento económico y ambiental, podríamos estar en presencia de formas de gobernanza y autonomía relacional (Ulloa, 2011), que desplazan la tradicional centralidad de la dimensión económica y contrarrestan la dependencia de un único encargado del museo.

En segundo lugar, se observa que la dimensión económica se propone como una propiedad emergente en la gestión de las dimensiones genéricas y sinérgicas, entendiéndola como el resultado de la administración de lo que se tiene para satisfacer sus necesidades. Lo que nos permite identificar una correlación inversa, entre el 
sistema económico actual y la propuesta de sostenibilidad situada. Cuanto más se incentiva una economía basada en la competencia, la hegemonía y la fragmentación entre la humanidad y la vida, se actúa en detrimento de la comunidad, la diversidad cultural y natural. Por el contrario, si se incentiva el reconocimiento de valores locales, dichos mecanismos destructores perderán fuerza y emergerán nuevos paradigmas alternativos, relacionales y creativos, para enfrentar tantas realidades como valores existan.

Lo anterior se encuentra en las experiencias estudiadas, tal como se expuso en las sinergias de reciprocidad, colaboración y pertinencia, está emergiendo nuevamente una cultura ecológica, muy rica en cuanto a su complejidad y diversidad, en modelos holísticos de percepción y uso de los recursos, confrontando la degradación ambiental y social, a partir, de la reinterpretación y utilización de prácticas ancestrales, caracterizadas por su complementariedad entre espacios físicos y ciclos naturales, como ordenamiento ecológico de procesos productivos y simbólicos (Leff, 2000), mismas prácticas que se vuelven parte del patrimonio natural y cultural, donde la naturaleza es tanto recurso económico como patrimonial.

Desde esta perspectiva, se puede diseñar una visión de sostenibilidad local, integradora y alcanzable, donde los procesos de enraizamiento e interpretación de conceptos racionalizados e institucionalizados, se reconfiguran práctica y simbólicamente por medio de mecanismos culturales propios. Así, se podría concebir el museo como una herramienta política para las comunidades en la defensa de su patrimonio y territorio (Varine, 2012).

Como ya lo decía Ehrenfeld, (2008), citado por Escobar, "la clave de la sustentabilidad radica en las verdades prácticas que cada uno de nosotros descubre en la vida cotidiana y que contribuyen a las actividades colectivas de nuestra cultura” (en Escobar 2016, p.142). Por lo cual, el museo se convierte en un espacio potencial generador y visibilizador de relaciones de poder, destacando su potencial creativo para autodefinir la sostenibilidad, y no, para implementar un deber ser impuesto, "lo que equivale a decir que la cultura es a la vez socialmente determinada y determinante, a la vez estructurada y estructurante” (Bassand, 1981, en Giménez 1996).

Finalmente, se destaca la potencialidad de una sostenibilidad que reconozca la cultura como un pilar fundamental, donde como lo describe Davis (2011) "la diversidad de la vida en todas sus manifestaciones, biológicas, culturales y lingüísticas, están interrelacionadas (y probablemente evolucionan conjuntamente) dentro de un complejo sistema de adaptación socio ecológica”. Con esto se hace referencia, además, a que cada museo es un caso único al igual que su contexto, por lo tanto, no se busca teorizar las diversas realidades para verificarlas en lo empírico, por el contrario, se busca un proceso de construcción y validación entre museo y comunidad, en donde se resguardan valores, más que verdades (Goodman en DeCarli, 2004).

\section{Conclusiones}

Se caracterizó a través de dimensiones generales y dimensiones sinérgicas, tres de los museos comunitarios o de pequeña escala de la Región de los Ríos, lo que nos permitió identificar las condiciones sociales, culturales, ambientales y económicas de sus contextos, y a partir de éstas, profundizar el estudio centrado en las relaciones socio-naturales, para así comprender y relevar aspectos cualitativos de aquello que propicia la sostenibilidad.

A través del análisis de las dimensiones generales, se identifica que las dificultades en la proyección de los museos, se relaciona en primera instancia con aspectos materiales y su precariedad en las diferentes áreas de trabajo. Sin embargo, a través del análisis de las dimensiones sinérgicas, se identifican valores no materiales que emergen en la ausencia de los primeros, así como conflictos de los que el museo es parte, enfrenta y/o es testigo. Por lo tanto el reto fundamental frente a la sostenibilidad, es cómo se propicia la conexión de los museos con los habitantes de las comunidades, y la agencia de éste frente a las problemáticas del territorio al que pertenecen. Sin embargo, en un espectro más amplio, el reto también está en la forma como estas relaciones se legitiman y visibilizan, frente a las visiones instrumentalizadas de la sostenibilidad. 
El delinear valores como la reciprocidad, la colaboración y la pertinencia, acentúa el potencial creativo que tienen estos museos para indagar en el proceso histórico de creación, mediación y condicionamiento de la materialidad en determinados territorios y comunidades, para el planteamiento de teorías críticas de su realidad, a través de propuestas participativas de transformación hacía modelos sostenibles y situados, convirtiéndose en un mecanismo de resistencia, de generación de conciencia y construcción frente a la globalización y sus implicancias en los distintos niveles, partiendo esencialmente de la cultura.

La evidencia presentada nos permite concluir que la sostenibilidad situada, se refiere a cualidades de los sistemas vivos, y que los museos estudiados dentro del enfoque de la nueva museología, representan la heterogeneidad, los principios y prácticas que dilucidaban las recomendaciones de los museos integrales. Por lo tanto, hablamos de una función social que se ha desarrollado de manera empírica, sin embargo, perfectible, no en el sentido de llegar a un deber ser, sino, en el sentido de reconocer que por destacables que sean las experiencias, su dependencia de seres sociales también ilustra la fragilidad de los mismos.

\section{Agradecimientos}

Este artículo es resultado del Proyecto EU LAC MUSEUSM No 693669 que ha recibido fondos del Programa de Investigación e Innovación H2020 de la Comunidad Europea.

Además se adscribe al Núcleo Milenio Energía y Sociedad, Valdivia, Chile.

\section{Notas}

\footnotetext{
${ }^{1}$ Más información sobre la Red de Museos en http://www.museosregiondelosrios.cl/index.php

${ }^{2}$ Museo: Institución permanente, sin fines de lucro al servicio de la sociedad y su desarrollo, abierto al público, que adquiere, conserva, investiga, comunica y exhibe el patrimonio tangible e intangible de la humanidad y se ambienta con propósito de educación, estudio y recreación. (ICOM, adaptado en Viena 2007)

${ }^{3}$ En este contexto los cabildos se refieren a reuniones de discusión frente a un tema o situación, donde participa la comunidad para reflexionar y dejar manifiesto algunas declaraciones o acciones que se podrían llevar a cabo.
}

\section{Bibliografía}

Basso, I. y Schwerter, C. (2009). Reconstrucción de la memoria histórica del barrio La Aguada. Consejo Nacional de la Cultura y las Artes, Región de Los Ríos. En Plan Municipal de Cultural Comuna de Corral 20162020 .

Chagas, M. (2008). Museos, educación y movimientos sociales: solo la antropofagia nos une. En Museos, educación y juventud.(pp.14-18) Bogotá, Colombia: Ministerio de Cultura

Bize, C. y Weil, K. (2017). Sobre la "Mesa de Santiago” de 1972?y la función social del museo en la actualidad. Informe de avance, Proyecto EULAC Museums Horizonte 2020, $n^{\circ} 693669$.

Davis, P. (2011). Ecomuseum: A Sense of Place. London, England: Continuum International Publishing.

DeCarli, G. (2003). Vigencia de la Nueva Museología en América Latina: conceptos y modelos. Revista ABRA,24(33) ,55-75. Recuperado de https://www.revistas.una.ac.cr/index.php/abra/article/view/4207

DeCarli, G. (2004). Un Museo sostenible: museo y comunidad en la preservación activa de su patrimonio. San José Costa Rica: Oficina de la UNESCO para América Central.

Desvallées, A. y Mairesse, F. (2010). Conceptos claves de museología. ICOM International Committee for Museology 
(ICOFOM). Paris, Francia: Armand Colin. Recuperado de: http://icom.museum/fileadmin/user_upload/pdf/ Key_Concepts_of_Museology/Museologie_Espagnol_BD.pdf

DeVarine, H. (2012) Reflexiones a 40 años de la Mesa de Santiago.Revista Museos (31), 4-6. Recuperado de: https://www.patrimoniocultural.gob.cl/614/articles-6893_archivo_01.pdf

Dos Santos, P. (2012). La Mesa de Santiago para pensar un futuro.En Resoluciones de "La Mesa Redonda: La importancia y el desarrollo de los museos en el mundo contemporáneo”, organizado por UNESCO- ICOM, Santiago de Chile, 31 de Mayo de 1972.

Boyer, R. H., Peterson, N. D., Arora, P. y Caldwell, K. (2016). Five Approaches to Social Sustainability and an Integrated Way Forward.RevistaSostenibilidad,8 (9), 878.Doi 10.3390/su8090878

Escobar, A. (2016). Autonomía y Diseño: La realización de lo comunal, Popayán, Colombia: Universidad del Cauca.

Giménez. G. (1996). Territorio y cultura. Estudios sobre las Culturas Contemporáneas.2 (4), 9-30.Recuperado de http://www.redalyc.org/pdf/316/31600402.pdf

Hajer, M. (1995). The politics of environmental discourse: ecological modernization and the policy process. Oxford: Clarendon Press.Doi 10.1093 / 019829333X.001.0001

ICOM (11 de septiembre de 2018). ICOM crea un nuevo grupo de trabajo sobre la sostenibilidad. Recuperado de: https://icom.museum/es/news/el-icom-crea-un-nuevo-grupo-de-trabajo-sobre-la-sostenibilidad/

Laumonier, I. (1993). Museo y Sociedad. Buenos Aires, Argentina: Cetro Editor de América Latina.

Leff, E. (2000). Espacio, lugar y tiempo: la reapropiación social de la naturaleza y la construcción local de la racionalidad ambiental. Desenvolvimento e Meio Ambiente (1), 57-69.Doi 10.5380/dma.v1i0.3057

Mallmann, C. A., Max-Neef, M.A. y Aguirre, R.A. (1978). La Sinergia Humana como Fundamento Ético y Estético del Desarrollo (Una mostración sinfónica). Bariloche, Argentina:Fundación Bariloche.

Manzini. E. (2015). Cuando todos diseñan. Una introducción al diseño para la innovación social. Madrid, España: Experimenta Editorial.

Marsal, D. (comp.) (2012). Hecho en Chile. Reflexiones en torno al patrimonio cultural. Santiago, Chile. Andros Impresores.

Max-Neef, M. A., Elizalde, A. yHopenhaim, M. (1986). Desarrollo a Escala Humana: conceptos, aplicaciones y reflexiones. Barcelona, España: Icaria Editorial.

Max-Neef, M. A., (2004). Fundamentos de la Transdisciplinaridad. Universidad Austral de Chile. Valdivia. Chile.

Mostny, G. (1975). Los Museos de Chile. Coleccioìn Nosotros los Chilenos. Santiago, Chile: Direccioìn de Bibliotecas, Archivos y Museos (DIBAM).

Pierri, N. (coord.) (2005). ¿Sustentabilidad? Desacuerdos sobre el desarrollo sustentable, Colección América Latina y el Nuevo Orden Mundial. México: Miguel Ángel Porrua, UAZ, Cámara de Diputados LIX Legislatura,

Quijano, A. (2000). Colonialidad del poder, eurocentrismo y América Latina. Lander, E. (comp).La colonialidad del saber: eurocentrismo y ciencias sociales. Perspectivas Latinoamericanas, 201-242. Buenos Aires, Argentina: CLACSO, Consejo Latinoamericano de Ciencias Sociales 
Quiroga, R. (2012). Para forjar sociedades sustentables. Polis Revista Latinoamericana,(5),1-17.Doihttp:// dx.doi.org/10.32735/S0718-6568/2003-N5-229

Pérez, A. (2016). Marco conceptual común en sostenibilidad de las instituciones y procesos museísticos iberoamericanos. Programa Ibermuseos. Línea de Acción Sostenibilidad de las Instituciones y Procesos Museísticos Iberoamericanos. Brasilia, Brasil: Instituto Brasileiro de Museus - Ibram.

Spaargaren, G., y Mol, A. (1992). Sociology, environment, and modernity: Ecological modernization as a theory of social change. Society \& natural resources, 5(4), 323-344.Doi 10.1080/08941929209380797

Sutton, S. (2015). Environmental sustainability at historic cities and museums. Lanham, United States of America: Rowman \& Littlefield.

Ulloa, A. (2011). Políticas globales del cambio climático: nuevas geopolíticas del conocimiento y sus efectos en territorios indígenas. Buenos Aires, Argentina: Consejo Latinoamericano de Ciencias Sociales (CLACSO).

Varine, H. (2012). Alrededor de la mesa redonda de Santiago, intervención en ocasión de una reunión realizada en São Paulo comienzo de los años 2000. En Mesa Redonda vol. I.

Weil, K., Fúquene, L., Blanco, G., Urbina, S. y Bize, C.. (2017). Caracterización de la Red de Museos de la Región de Los Ríos y selección de casos de estudios. Informe de avance, año 1. Proyecto EULAC Museums Horizonte 2020, $n^{\circ} 693669$. 